\title{
Effect of Mo/P Ratios on Dimensions: Syntheses, Structures and Properties of Three New Molybdophosphates
}

Haonan Liu, ${ }^{a}$ Hongping $W u,{ }^{a}$ Hongwei $Y u,{ }^{a}{ }^{*}$ Zhanggui Hu, ${ }^{a}{ }^{*}$ Jiyang Wang ${ }^{a}$ and Yicheng $W u^{a}$

${ }^{a}$ Tianjin Key Laboratory of Functional Crystal Materials, Institute of Functional Crystal, Tianjin University of Technology, Tianjin 300384, China.

To whom correspondence should be addressed. E-mail: hwyu15@gmail.com (Hongwei Yu).

\section{CONTENTS}

1. Table S1 (Atomic coordinates, displacement parameters and BVS)................................S2

2. Table $S 2$ (Selected bond distances and angles) ...............................................................S3

3. Table S3 (Bond valence sums) ..............................................................................................S7

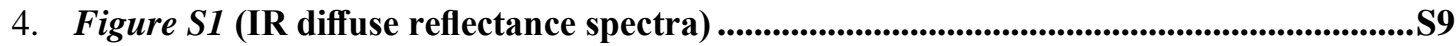

5. Table $S 4$ (The assignments of the infrared absorption peaks) ..........................................S9

6. Figure $S 2$ (UV-VIS-NIR diffuse reflectance spectra) .....................................................S9

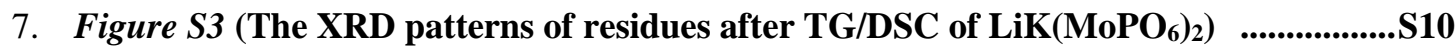

8. Figure $S 4$ (The XRD patterns of residues after TG/DSC of $\left.\mathrm{Sr}\left(\mathrm{MoPO}_{6}\right)_{2}\right)$.....................S10

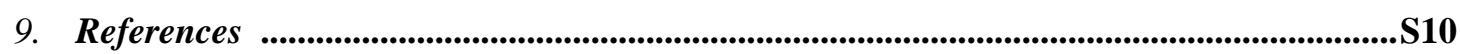


Table S1. Atomic coordinates and equivalent isotropic displacement parameters for $\mathrm{Cs}_{3} \mathrm{Mo}_{12} \mathrm{PO}_{40}$, $\mathrm{LiK}\left(\mathrm{MoPO}_{6}\right)_{2}$ and $\mathrm{Sr}\left(\mathrm{MoPO}_{6}\right)_{2}$.

$\mathrm{Cs}_{3} \mathrm{Mo}_{12} \mathrm{PO}_{40}$

\begin{tabular}{cccccc}
\hline Atom & $\mathrm{x}$ & $\mathrm{y}$ & $\mathrm{z}$ & $\mathrm{U}(\mathrm{eq})$ & $\mathrm{BVS}$ \\
\hline $\mathrm{Cs}(1)$ & 2500 & 7500 & 2500 & $19(1)$ & 1.2081 \\
$\mathrm{Mo}(1)$ & $371(1)$ & $10371(1)$ & $2588(1)$ & $13(1)$ & 5.9311 \\
$\mathrm{P}(1)$ & 2500 & 12500 & 2500 & $7(1)$ & 4.9121 \\
$\mathrm{O}(1)$ & $1247(1)$ & $9653(2)$ & $3753(1)$ & $14(1)$ & 1.9909 \\
$\mathrm{O}(2)$ & $1749(2)$ & $11749(2)$ & $3251(2)$ & $10(1)$ & 1.9403 \\
$\mathrm{O}(3)$ & $1542(1)$ & $9984(2)$ & $1542(1)$ & $16(1)$ & 2.0316 \\
$\mathrm{O}(4)$ & $-614(2)$ & $9386(2)$ & $2318(2)$ & $20(1)$ & 1.9733 \\
\hline
\end{tabular}

$\mathrm{LiK}\left(\mathrm{MoPO}_{6}\right)_{2}$

\begin{tabular}{cccccc}
\hline Atom & $\mathrm{x}$ & $\mathrm{y}$ & $\mathrm{z}$ & $\mathrm{U}(\mathrm{eq})$ & $\mathrm{BVS}$ \\
\hline $\mathrm{Li}(1)$ & $230(50)$ & $8190(30)$ & $9690(20)$ & $64(9)$ & 0.89 \\
$\mathrm{~K}(1)$ & 5000 & 10000 & 10000 & $48(1)$ & 0.74 \\
$\mathrm{Mo}(1)$ & $7716(1)$ & $10342(1)$ & $6849(1)$ & $9(1)$ & 6.01 \\
$\mathrm{P}(1)$ & $2610(2)$ & $9503(1)$ & $6603(1)$ & $7(1)$ & 4.95 \\
$\mathrm{O}(1)$ & $4624(5)$ & $10655(4)$ & $6928(3)$ & $13(1)$ & 2.01 \\
$\mathrm{O}(2)$ & $727(4)$ & $10842(4)$ & $6491(3)$ & $11(1)$ & 2.11 \\
$\mathrm{O}(3)$ & $2388(4)$ & $8215(4)$ & $7819(3)$ & $12(1)$ & 1.93 \\
$\mathrm{O}(4)$ & $2704(4)$ & $8480(4)$ & $5233(3)$ & $12(1)$ & 1.96 \\
$\mathrm{O}(5)$ & $8394(5)$ & $9932(5)$ & $8585(3)$ & $18(1)$ & 2.15 \\
$\mathrm{O}(6)$ & $7590(5)$ & $8241(4)$ & $6154(3)$ & $18(1)$ & 1.81 \\
\hline
\end{tabular}

$\mathrm{Sr}\left(\mathrm{MoPO}_{6}\right)_{2}$

\begin{tabular}{cccccc}
\hline Atom & $\mathrm{x}$ & $\mathrm{y}$ & $\mathrm{z}$ & $\mathrm{U}(\mathrm{eq})$ & $\mathrm{BVS}$ \\
\hline $\mathrm{Sr}(1)$ & $4875(1)$ & $3720(1)$ & $2610(1)$ & $13(1)$ & 1.93 \\
$\mathrm{Mo}(1)$ & $2425(1)$ & $1282(1)$ & $4056(1)$ & $6(1)$ & 6.02 \\
$\mathrm{Mo}(2)$ & $2429(1)$ & $6144(1)$ & $4010(1)$ & $6(1)$ & 6.06 \\
$\mathrm{P}(1)$ & $2587(1)$ & $3767(1)$ & $5437(1)$ & $6(1)$ & 4.96 \\
$\mathrm{P}(2)$ & $2593(1)$ & $5679(1)$ & $1109(1)$ & $7(1)$ & 4.98 \\
$\mathrm{O}(1)$ & $4455(3)$ & $4175(2)$ & $6223(2)$ & $9(1)$ & 1.99 \\
$\mathrm{O}(2)$ & $562(4)$ & $6170(2)$ & $547(2)$ & $11(1)$ & 2.05 \\
$\mathrm{O}(3)$ & $2808(4)$ & $6340(2)$ & $5441(2)$ & $12(1)$ & 1.95 \\
$\mathrm{O}(4)$ & $587(3)$ & $4101(2)$ & $6059(2)$ & $11(1)$ & 2.13 \\
$\mathrm{O}(5)$ & $2554(4)$ & $80(2)$ & $3319(2)$ & $12(1)$ & 1.99 \\
$\mathrm{O}(6)$ & $3153(3)$ & $4562(2)$ & $610(2)$ & $10(1)$ & 2.00 \\
$\mathrm{O}(7)$ & $2614(3)$ & $4342(2)$ & $4280(2)$ & $9(1)$ & 1.96 \\
\hline
\end{tabular}




\begin{tabular}{cccccc}
\hline $\mathrm{O}(8)$ & $2343(3)$ & $5432(2)$ & $2373(2)$ & $10(1)$ & 2.08 \\
$\mathrm{O}(9)$ & $4369(4)$ & $6519(2)$ & $952(2)$ & $12(1)$ & 1.96 \\
$\mathrm{O}(10)$ & $2706(3)$ & $2526(2)$ & $5318(2)$ & $10(1)$ & 1.85 \\
$\mathrm{O}(11)$ & $2606(4)$ & $7437(2)$ & $3468(2)$ & $14(1)$ & 1.98 \\
$\mathrm{O}(12)$ & $2114(4)$ & $2246(2)$ & $3005(2)$ & $14(1)$ & 2.00 \\
\hline
\end{tabular}

Table S2. Selected bond distances $(\AA)$ and angles $\left(^{\circ}\right)$ for $\mathrm{Cs}_{3} \mathrm{Mo}_{12} \mathrm{PO}_{40}, \mathrm{LiK}\left(\mathrm{MoPO}_{6}\right)_{2}$ and $\mathrm{Sr}\left(\mathrm{MoPO}_{6}\right)_{2}$.

$\mathrm{Cs}_{3} \mathrm{Mo}_{12} \mathrm{PO}_{40}$

\begin{tabular}{|c|c|c|c|}
\hline \multicolumn{4}{|l|}{ Distances $(\AA)$} \\
\hline $\mathrm{Cs}(1)-\mathrm{O}(4) \# 1$ & $3.168(3)$ & $\mathrm{Cs}(1)-\mathrm{O}(3) \# 5$ & $3.353(2)$ \\
\hline $\mathrm{Cs}(1)-\mathrm{O}(4) \# 2$ & $3.168(3)$ & $\mathrm{Mo}(1)-\mathrm{O}(4)$ & $1.681(3)$ \\
\hline $\mathrm{Cs}(1)-\mathrm{O}(4) \# 3$ & $3.168(3)$ & $\mathrm{Mo}(1)-\mathrm{O}(3) \# 8$ & $1.9158(6)$ \\
\hline $\mathrm{Cs}(1)-\mathrm{O}(4) \# 4$ & $3.168(3)$ & $\mathrm{Mo}(1)-\mathrm{O}(3)$ & $1.9158(6)$ \\
\hline $\mathrm{Cs}(1)-\mathrm{O}(1) \# 5$ & $3.304(2)$ & $\mathrm{Mo}(1)-\mathrm{O}(1) \# 8$ & $1.9257(9)$ \\
\hline $\mathrm{Cs}(1)-\mathrm{O}(1) \# 6$ & $3.304(2)$ & $\mathrm{Mo}(1)-\mathrm{O}(1)$ & $1.9257(9)$ \\
\hline $\mathrm{Cs}(1)-\mathrm{O}(1)$ & $3.304(2)$ & $\mathrm{Mo}(1)-\mathrm{O}(2)$ & $2.439(2)$ \\
\hline $\mathrm{Cs}(1)-\mathrm{O}(1) \# 7$ & $3.304(2)$ & $\mathrm{P}(1)-\mathrm{O}(2) \# 10$ & $1.541(3)$ \\
\hline $\mathrm{Cs}(1)-\mathrm{O}(3) \# 6$ & $3.353(2)$ & $\mathrm{P}(1)-\mathrm{O}(2) \# 11$ & $1.541(3)$ \\
\hline $\mathrm{Cs}(1)-\mathrm{O}(3) \# 7$ & $3.353(2)$ & $\mathrm{P}(1)-\mathrm{O}(2) \# 9$ & $1.541(3)$ \\
\hline $\mathrm{Cs}(1)-\mathrm{O}(3)$ & $3.353(2$ & $\mathrm{P}(1)-\mathrm{O}(2)$ & $1.541(3)$ \\
\hline \multicolumn{4}{|l|}{ Angles ( ${ }^{\circ}$ ) } \\
\hline $\mathrm{O}(4) \# 1-\mathrm{Cs}(1)-\mathrm{O}(4) \# 2$ & $172.20(8)$ & $\mathrm{O}(3) \# 6-\mathrm{Cs}(1)-\mathrm{O}(3) \# 7$ & $140.40(5)$ \\
\hline $\mathrm{O}(4) \# 1-\mathrm{Cs}(1)-\mathrm{O}(4) \# 3$ & $90.265(6)$ & $\mathrm{O}(4) \# 1-\mathrm{Cs}(1)-\mathrm{O}(3)$ & $57.48(5)$ \\
\hline $\mathrm{O}(4) \# 2-\mathrm{Cs}(1)-\mathrm{O}(4) \# 3$ & $90.265(6)$ & $\mathrm{O}(4) \# 2-\mathrm{Cs}(1)-\mathrm{O}(3)$ & $114.72(6)$ \\
\hline $\mathrm{O}(4) \# 1-\mathrm{Cs}(1)-\mathrm{O}(4) \# 4$ & $90.265(6)$ & $\mathrm{O}(4) \# 3-\mathrm{Cs}(1)-\mathrm{O}(3)$ & $93.42(4)$ \\
\hline $\mathrm{O}(4) \# 2-\mathrm{Cs}(1)-\mathrm{O}(4) \# 4$ & $90.265(6)$ & $\mathrm{O}(4) \# 4-\mathrm{Cs}(1)-\mathrm{O}(3)$ & $93.42(4)$ \\
\hline $\mathrm{O}(4) \# 3-\mathrm{Cs}(1)-\mathrm{O}(4) \# 4$ & $172.20(8)$ & $\mathrm{O}(1) \# 5-\mathrm{Cs}(1)-\mathrm{O}(3)$ & $111.91(5)$ \\
\hline $\mathrm{O}(4) \# 1-\mathrm{Cs}(1)-\mathrm{O}(1) \# 5$ & $54.43(5)$ & $\mathrm{O}(1) \# 6-\mathrm{Cs}(1)-\mathrm{O}(3)$ & $47.34(3)$ \\
\hline $\mathrm{O}(4) \# 2-\mathrm{Cs}(1)-\mathrm{O}(1) \# 5$ & $133.37(5)$ & $\mathrm{O}(1)-\mathrm{Cs}(1)-\mathrm{O}(3)$ & $47.34(3)$ \\
\hline $\mathrm{O}(4) \# 3-\mathrm{Cs}(1)-\mathrm{O}(1) \# 5$ & $86.99(3)$ & $\mathrm{O}(1) \# 7-\mathrm{Cs}(1)-\mathrm{O}(3)$ & $169.15(5)$ \\
\hline $\mathrm{O}(4) \# 4-\mathrm{Cs}(1)-\mathrm{O}(1) \# 5$ & $86.99(3)$ & $\mathrm{O}(3) \# 6-\mathrm{Cs}(1)-\mathrm{O}(3)$ & $57.24(7)$ \\
\hline $\mathrm{O}(4) \# 1-\mathrm{Cs}(1)-\mathrm{O}(1) \# 6$ & $86.99(3)$ & $\mathrm{O}(3) \# 7-\mathrm{Cs}(1)-\mathrm{O}(3)$ & $140.40(5)$ \\
\hline $\mathrm{O}(4) \# 2-\mathrm{Cs}(1)-\mathrm{O}(1) \# 6$ & $86.99(3)$ & $\mathrm{O}(4) \# 1-\mathrm{Cs}(1)-\mathrm{O}(3) \# 5$ & $93.42(4)$ \\
\hline $\mathrm{O}(4) \# 3-\mathrm{Cs}(1)-\mathrm{O}(1) \# 6$ & $54.43(5)$ & $\mathrm{O}(4) \# 2-\mathrm{Cs}(1)-\mathrm{O}(3) \# 5$ & $93.42(4)$ \\
\hline $\mathrm{O}(4) \# 4-\mathrm{Cs}(1)-\mathrm{O}(1) \# 6$ & $133.37(5)$ & $\mathrm{O}(4) \# 3-\mathrm{Cs}(1)-\mathrm{O}(3) \# 5$ & $114.72(6)$ \\
\hline $\mathrm{O}(1) \# 5-\mathrm{Cs}(1)-\mathrm{O}(1) \# 6$ & $126.58(4)$ & $\mathrm{O}(4) \# 4-\mathrm{Cs}(1)-\mathrm{O}(3) \# 5$ & $57.48(5)$ \\
\hline $\mathrm{O}(4) \# 1-\mathrm{Cs}(1)-\mathrm{O}(1)$ & $86.99(3)$ & $\mathrm{O}(1) \# 5-\mathrm{Cs}(1)-\mathrm{O}(3) \# 5$ & $47.34(3)$ \\
\hline $\mathrm{O}(4) \# 2-\mathrm{Cs}(1)-\mathrm{O}(1)$ & $86.99(3)$ & $\mathrm{O}(1) \# 6-\mathrm{Cs}(1)-\mathrm{O}(3) \# 5$ & $169.15(5)$ \\
\hline $\mathrm{O}(4) \# 3-\mathrm{Cs}(1)-\mathrm{O}(1)$ & $133.37(5)$ & $\mathrm{O}(1)-\mathrm{Cs}(1)-\mathrm{O}(3) \# 5$ & $111.91(5)$ \\
\hline $\mathrm{O}(4) \# 4-\mathrm{Cs}(1)-\mathrm{O}(1)$ & $54.43(5)$ & $\mathrm{O}(1) \# 7-\mathrm{Cs}(1)-\mathrm{O}(3) \# 5$ & $47.34(3)$ \\
\hline $\mathrm{O}(1) \# 5-\mathrm{Cs}(1)-\mathrm{O}(1)$ & $126.58(4)$ & $\mathrm{O}(3) \# 6-\mathrm{Cs}(1)-\mathrm{O}(3) \# 5$ & $140.40(5)$ \\
\hline $\mathrm{O}(1) \# 6-\mathrm{Cs}(1)-\mathrm{O}(1)$ & $78.94(7)$ & $\mathrm{O}(3) \# 7-\mathrm{Cs}(1)-\mathrm{O}(3) \# 5$ & $57.24(7)$ \\
\hline $\mathrm{O}(4) \# 1-\mathrm{Cs}(1)-\mathrm{O}(1) \# 7$ & $133.37(5)$ & $\mathrm{O}(3)-\mathrm{Cs}(1)-\mathrm{O}(3) \# 5$ & $140.40(5)$ \\
\hline
\end{tabular}




\begin{tabular}{llll}
\hline $\mathrm{O}(4) \# 2-\mathrm{Cs}(1)-\mathrm{O}(1) \# 7$ & $54.43(5)$ & $\mathrm{O}(4)-\mathrm{Mo}(1)-\mathrm{O}(3) \# 8$ & $102.32(9)$ \\
$\mathrm{O}(4) \# 3-\mathrm{Cs}(1)-\mathrm{O}(1) \# 7$ & $86.99(3)$ & $\mathrm{O}(4)-\mathrm{Mo}(1)-\mathrm{O}(3)$ & $102.32(9)$ \\
$\mathrm{O}(4) \# 4-\mathrm{Cs}(1)-\mathrm{O}(1) \# 7$ & $86.99(3)$ & $\mathrm{O}(3) \# 8-\mathrm{Mo}(1)-\mathrm{O}(3)$ & $85.87(13)$ \\
$\mathrm{O}(1) \# 5-\mathrm{Cs}(1)-\mathrm{O}(1) \# 7$ & $78.94(7)$ & $\mathrm{O}(4)-\mathrm{Mo}(1)-\mathrm{O}(1) \# 8$ & $101.74(8)$ \\
$\mathrm{O}(1) \# 6-\mathrm{Cs}(1)-\mathrm{O}(1) \# 7$ & $126.58(4)$ & $\mathrm{O}(3) \# 8-\mathrm{Mo}(1)-\mathrm{O}(1) \# 8$ & $88.18(9)$ \\
$\mathrm{O}(1)-\mathrm{Cs}(1)-\mathrm{O}(1) \# 7$ & $126.58(4)$ & $\mathrm{O}(3)-\mathrm{Mo}(1)-\mathrm{O}(1) \# 8$ & $155.91(8)$ \\
$\mathrm{O}(4) \# 1-\mathrm{Cs}(1)-\mathrm{O}(3) \# 6$ & $114.72(6)$ & $\mathrm{O}(4)-\mathrm{Mo}(1)-\mathrm{O}(1)$ & $101.74(8)$ \\
$\mathrm{O}(4) \# 2-\mathrm{Cs}(1)-\mathrm{O}(3) \# 6$ & $57.48(5)$ & $\mathrm{O}(3) \# 8-\mathrm{Mo}(1)-\mathrm{O}(1)$ & $155.91(8)$ \\
$\mathrm{O}(4) \# 3-\mathrm{Cs}(1)-\mathrm{O}(3) \# 6$ & $93.42(4)$ & $\mathrm{O}(3)-\mathrm{Mo}(1)-\mathrm{O}(1)$ & $88.18(9)$ \\
$\mathrm{O}(4) \# 4-\mathrm{Cs}(1)-\mathrm{O}(3) \# 6$ & $93.42(4)$ & $\mathrm{O}(1) \# 8-\mathrm{Mo}(1)-\mathrm{O}(1)$ & $87.82(13)$ \\
$\mathrm{O}(1) \# 5-\mathrm{Cs}(1)-\mathrm{O}(3) \# 6$ & $169.15(5)$ & $\mathrm{O}(4)-\mathrm{Mo}(1)-\mathrm{O}(2)$ & $172.16(10)$ \\
$\mathrm{O}(1) \# 6-\mathrm{Cs}(1)-\mathrm{O}(3) \# 6$ & $47.34(3)$ & $\mathrm{O}(3) \# 8-\mathrm{Mo}(1)-\mathrm{O}(2)$ & $83.34(8)$ \\
$\mathrm{O}(1)-\mathrm{Cs}(1)-\mathrm{O}(3) \# 6$ & $47.34(3)$ & $\mathrm{O}(3)-\mathrm{Mo}(1)-\mathrm{O}(2)$ & $83.34(8)$ \\
$\mathrm{O}(1) \# 7-\mathrm{Cs}(1)-\mathrm{O}(3) \# 6$ & $111.91(5)$ & $\mathrm{O}(1) \# 8-\mathrm{Mo}(1)-\mathrm{O}(2)$ & $72.79(7)$ \\
$\mathrm{O}(4) \# 1-\mathrm{Cs}(1)-\mathrm{O}(3) \# 7$ & $93.42(4)$ & $\mathrm{O}(1)-\mathrm{Mo}(1)-\mathrm{O}(2)$ & $72.79(7)$ \\
$\mathrm{O}(4) \# 2-\mathrm{Cs}(1)-\mathrm{O}(3) \# 7$ & $93.42(4)$ & $\mathrm{O}(2) \# 10-\mathrm{P}(1)-\mathrm{O}(2) \# 11$ & $109.471(1)$ \\
$\mathrm{O}(4) \# 3-\mathrm{Cs}(1)-\mathrm{O}(3) \# 7$ & $57.48(5)$ & $\mathrm{O}(2) \# 10-\mathrm{P}(1)-\mathrm{O}(2) \# 9$ & 109.5 \\
$\mathrm{O}(4) \# 4-\mathrm{Cs}(1)-\mathrm{O}(3) \# 7$ & $114.72(6)$ & $\mathrm{O}(2) \# 11-\mathrm{P}(1)-\mathrm{O}(2) \# 9$ & 109.5 \\
$\mathrm{O}(1) \# 5-\mathrm{Cs}(1)-\mathrm{O}(3) \# 7$ & $47.34(3)$ & $\mathrm{O}(2) \# 10-\mathrm{P}(1)-\mathrm{O}(2)$ & 109.5 \\
$\mathrm{O}(1) \# 6-\mathrm{Cs}(1)-\mathrm{O}(3) \# 7$ & $111.91(5)$ & $\mathrm{O}(2) \# 11-\mathrm{P}(1)-\mathrm{O}(2)$ & 109.5 \\
$\mathrm{O}(1)-\mathrm{Cs}(1)-\mathrm{O}(3) \# 7$ & $169.15(5)$ & $\mathrm{O}(2) \# 9-\mathrm{P}(1)-\mathrm{O}(2)$ & $109.471(1)$ \\
$\mathrm{O}(1) \# 7-\mathrm{Cs}(1)-\mathrm{O}(3) \# 7$ & $47.34(3)$ & & \\
\hline
\end{tabular}

\begin{tabular}{llll}
$\mathrm{LiK}\left(\mathrm{MoPO}_{6}\right)_{2}$ & & & \\
\hline $\mathrm{Distances}(\AA)$ & & & \\
$\mathrm{Li}(1)-\mathrm{O}(5) \# 1$ & $1.980(19)$ & $\mathrm{K}(1)-\mathrm{O}(1) \# 4$ & $2.994(3)$ \\
$\mathrm{Li}(1)-\mathrm{O}(4) \# 2$ & $2.02(2)$ & $\mathrm{K}(1)-\mathrm{O}(6) \# 2$ & $3.052(3)$ \\
$\mathrm{Li}(1)-\mathrm{O}(2) \# 3$ & $2.13(2)$ & $\mathrm{K}(1)-\mathrm{O}(6) \# 8$ & $3.052(3)$ \\
$\mathrm{Li}(1)-\mathrm{O}(5) \# 4$ & $2.27(2)$ & $\mathrm{Mo}(1)-\mathrm{O}(6)$ & $1.688(3)$ \\
$\mathrm{Li}(1)-\mathrm{O}(3)$ & $2.38(3)$ & $\mathrm{Mo}(1)-\mathrm{O}(5)$ & $1.717(3)$ \\
$\mathrm{Li}(1)-\mathrm{O}(6) \# 5$ & $2.54(4)$ & $\mathrm{Mo}(1)-\mathrm{O}(1)$ & $1.992(3)$ \\
$\mathrm{K}(1)-\mathrm{O}(5) \# 4$ & $2.672(3)$ & $\mathrm{Mo}(1)-\mathrm{O}(2) \# 9$ & $2.017(3)$ \\
$\mathrm{K}(1)-\mathrm{O}(5)$ & $2.672(3)$ & $\mathrm{Mo}(1)-\mathrm{O}(3) \# 8$ & $2.146(3)$ \\
$\mathrm{K}(1)-\mathrm{O}(3) \# 4$ & $2.870(3)$ & $\mathrm{Mo}(1)-\mathrm{O}(4) \# 10$ & $2.183(3)$ \\
$\mathrm{K}(1)-\mathrm{O}(3)$ & $2.870(3)$ & $\mathrm{P}(1)-\mathrm{O}(3)$ & $1.529(3)$ \\
$\mathrm{K}(1)-\mathrm{O}(4) \# 8$ & $2.975(3)$ & $\mathrm{P}(1)-\mathrm{O}(4)$ & $1.530(3)$ \\
$\mathrm{K}(1)-\mathrm{O}(4) \# 2$ & $2.975(3)$ & $\mathrm{P}(1)-\mathrm{O}(1)$ & $1.545(3)$ \\
$\mathrm{K}(1)-\mathrm{O}(1)$ & $2.994(3)$ & $\mathrm{P}(1)-\mathrm{O}(2)$ & $1.549(3)$ \\
$\mathrm{Angles}\left({ }^{\circ}\right)$ & & & \\
$\mathrm{O}(5) \# 1-\mathrm{Li}(1)-\mathrm{O}(4) \# 2$ & $159.6(18)$ & $\mathrm{O}(4) \# 2-\mathrm{K}(1)-\mathrm{O}(1) \# 4$ & $77.07(8)$ \\
$\mathrm{O}(5) \# 1-\mathrm{Li}(1)-\mathrm{O}(2) \# 3$ & $96.7(9)$ & $\mathrm{O}(1)-\mathrm{K}(1)-\mathrm{O}(1) \# 4$ & $180.000(1)$ \\
$\mathrm{O}(4) \# 2-\mathrm{Li}(1)-\mathrm{O}(2) \# 3$ & $79.5(7)$ & $\mathrm{O}(5) \# 4-\mathrm{K}(1)-\mathrm{O}(6) \# 2$ & $105.26(9)$ \\
$\mathrm{O}(5) \# 1-\mathrm{Li}(1)-\mathrm{O}(5) \# 4$ & $99.3(9)$ & $\mathrm{O}(5)-\mathrm{K}(1)-\mathrm{O}(6) \# 2$ & $74.74(9)$ \\
$\mathrm{O}(4) \# 2-\mathrm{Li}(1)-\mathrm{O}(5) \# 4$ & $87.2(8)$ & $\mathrm{O}(3) \# 4-\mathrm{K}(1)-\mathrm{O}(6) \# 2$ & $80.75(8)$ \\
\hline & & &
\end{tabular}




\begin{tabular}{|c|c|c|c|}
\hline $\mathrm{O}(2) \# 3-\mathrm{Li}(1)-\mathrm{O}(5) \# 4$ & $163.2(12)$ & $\mathrm{O}(3)-\mathrm{K}(1)-\mathrm{O}(6) \# 2$ & $99.25(8)$ \\
\hline $\mathrm{O}(5) \# 1-\mathrm{Li}(1)-\mathrm{O}(3)$ & $86.5(10)$ & $\mathrm{O}(4) \# 8-\mathrm{K}(1)-\mathrm{O}(6) \# 2$ & $116.89(8)$ \\
\hline $\mathrm{O}(4) \# 2-\mathrm{Li}(1)-\mathrm{O}(3)$ & $73.1(9)$ & $\mathrm{O}(4) \# 2-\mathrm{K}(1)-\mathrm{O}(6) \# 2$ & $63.11(8)$ \\
\hline $\mathrm{O}(2) \# 3-\mathrm{Li}(1)-\mathrm{O}(3)$ & $75.8(7)$ & $\mathrm{O}(1)-\mathrm{K}(1)-\mathrm{O}(6) \# 2$ & $118.37(8)$ \\
\hline $\mathrm{O}(5) \# 4-\mathrm{Li}(1)-\mathrm{O}(3)$ & $110.1(14)$ & $\mathrm{O}(1) \# 4-\mathrm{K}(1)-\mathrm{O}(6) \# 2$ & $61.63(8)$ \\
\hline $\mathrm{O}(5) \# 1-\mathrm{Li}(1)-\mathrm{O}(6) \# 5$ & $100.4(12)$ & $\mathrm{O}(5) \# 4-\mathrm{K}(1)-\mathrm{O}(6) \# 8$ & $74.74(9)$ \\
\hline $\mathrm{O}(4) \# 2-\mathrm{Li}(1)-\mathrm{O}(6) \# 5$ & $98.4(11)$ & $\mathrm{O}(5)-\mathrm{K}(1)-\mathrm{O}(6) \# 8$ & $105.26(9)$ \\
\hline $\mathrm{O}(2) \# 3-\mathrm{Li}(1)-\mathrm{O}(6) \# 5$ & $77.9(11)$ & $\mathrm{O}(3) \# 4-\mathrm{K}(1)-\mathrm{O}(6) \# 8$ & $99.25(8)$ \\
\hline $\mathrm{O}(5) \# 4-\mathrm{Li}(1)-\mathrm{O}(6) \# 5$ & $94.2(9)$ & $\mathrm{O}(3)-\mathrm{K}(1)-\mathrm{O}(6) \# 8$ & $80.75(8)$ \\
\hline $\mathrm{O}(3)-\mathrm{Li}(1)-\mathrm{O}(6) \# 5$ & $153.4(11)$ & $\mathrm{O}(4) \# 8-\mathrm{K}(1)-\mathrm{O}(6) \# 8$ & $63.11(8)$ \\
\hline $\mathrm{O}(5) \# 4-\mathrm{K}(1)-\mathrm{O}(5)$ & $180.000(1)$ & $\mathrm{O}(4) \# 2-\mathrm{K}(1)-\mathrm{O}(6) \# 8$ & $116.89(8)$ \\
\hline $\mathrm{O}(5) \# 4-\mathrm{K}(1)-\mathrm{O}(3) \# 4$ & $93.13(9)$ & $\mathrm{O}(1)-\mathrm{K}(1)-\mathrm{O}(6) \# 8$ & $61.63(8)$ \\
\hline $\mathrm{O}(5)-\mathrm{K}(1)-\mathrm{O}(3) \# 4$ & $86.87(9)$ & $\mathrm{O}(1) \# 4-\mathrm{K}(1)-\mathrm{O}(6) \# 8$ & $118.37(8)$ \\
\hline $\mathrm{O}(5) \# 4-\mathrm{K}(1)-\mathrm{O}(3)$ & $86.87(9)$ & $\mathrm{O}(6) \# 2-\mathrm{K}(1)-\mathrm{O}(6) \# 8$ & $180.000(1)$ \\
\hline $\mathrm{O}(5)-\mathrm{K}(1)-\mathrm{O}(3)$ & $93.13(9)$ & $\mathrm{O}(6)-\mathrm{Mo}(1)-\mathrm{O}(5)$ & $102.95(16)$ \\
\hline $\mathrm{O}(3) \# 4-\mathrm{K}(1)-\mathrm{O}(3)$ & 180.0 & $\mathrm{O}(6)-\mathrm{Mo}(1)-\mathrm{O}(1)$ & $96.58(14)$ \\
\hline $\mathrm{O}(5) \# 4-\mathrm{K}(1)-\mathrm{O}(4) \# 8$ & $116.86(9)$ & $\mathrm{O}(5)-\mathrm{Mo}(1)-\mathrm{O}(1)$ & $97.76(14)$ \\
\hline $\mathrm{O}(5)-\mathrm{K}(1)-\mathrm{O}(4) \# 8$ & $63.14(9)$ & $\mathrm{O}(6)-\mathrm{Mo}(1)-\mathrm{O}(2) \# 9$ & $96.17(14)$ \\
\hline $\mathrm{O}(3) \# 4-\mathrm{K}(1)-\mathrm{O}(4) \# 8$ & $53.59(8)$ & $\mathrm{O}(5)-\mathrm{Mo}(1)-\mathrm{O}(2) \# 9$ & $92.86(14)$ \\
\hline $\mathrm{O}(3)-\mathrm{K}(1)-\mathrm{O}(4) \# 8$ & $126.41(8)$ & $\mathrm{O}(1)-\mathrm{Mo}(1)-\mathrm{O}(2) \# 9$ & $161.14(13)$ \\
\hline $\mathrm{O}(5) \# 4-\mathrm{K}(1)-\mathrm{O}(4) \# 2$ & $63.14(9)$ & $\mathrm{O}(6)-\mathrm{Mo}(1)-\mathrm{O}(3) \# 8$ & $164.95(13)$ \\
\hline $\mathrm{O}(5)-\mathrm{K}(1)-\mathrm{O}(4) \# 2$ & $116.86(9)$ & $\mathrm{O}(5)-\mathrm{Mo}(1)-\mathrm{O}(3) \# 8$ & $92.09(14)$ \\
\hline $\mathrm{O}(3) \# 4-\mathrm{K}(1)-\mathrm{O}(4) \# 2$ & $126.41(8)$ & $\mathrm{O}(1)-\mathrm{Mo}(1)-\mathrm{O}(3) \# 8$ & $80.45(12)$ \\
\hline $\mathrm{O}(3)-\mathrm{K}(1)-\mathrm{O}(4) \# 2$ & $53.59(8)$ & $\mathrm{O}(2) \# 9-\mathrm{Mo}(1)-\mathrm{O}(3) \# 8$ & $83.63(11)$ \\
\hline $\mathrm{O}(4) \# 8-\mathrm{K}(1)-\mathrm{O}(4) \# 2$ & 180.0 & $\mathrm{O}(6)-\mathrm{Mo}(1)-\mathrm{O}(4) \# 10$ & $90.15(13)$ \\
\hline $\mathrm{O}(5) \# 4-\mathrm{K}(1)-\mathrm{O}(1)$ & $121.09(9)$ & $\mathrm{O}(5)-\mathrm{Mo}(1)-\mathrm{O}(4) \# 10$ & $165.06(14)$ \\
\hline $\mathrm{O}(5)-\mathrm{K}(1)-\mathrm{O}(1)$ & $58.91(9)$ & $\mathrm{O}(1)-\mathrm{Mo}(1)-\mathrm{O}(4) \# 10$ & $87.69(12)$ \\
\hline $\mathrm{O}(3) \# 4-\mathrm{K}(1)-\mathrm{O}(1)$ & $129.54(8)$ & $\mathrm{O}(2) \# 9-\mathrm{Mo}(1)-\mathrm{O}(4) \# 10$ & $78.43(11)$ \\
\hline $\mathrm{O}(3)-\mathrm{K}(1)-\mathrm{O}(1)$ & $50.46(8)$ & $\mathrm{O}(3) \# 8-\mathrm{Mo}(1)-\mathrm{O}(4) \# 10$ & $75.03(11)$ \\
\hline $\mathrm{O}(4) \# 8-\mathrm{K}(1)-\mathrm{O}(1)$ & $77.07(8)$ & $\mathrm{O}(3)-\mathrm{P}(1)-\mathrm{O}(4)$ & $111.92(17)$ \\
\hline $\mathrm{O}(4) \# 2-\mathrm{K}(1)-\mathrm{O}(1)$ & $102.93(8)$ & $\mathrm{O}(3)-\mathrm{P}(1)-\mathrm{O}(1)$ & $108.95(17)$ \\
\hline $\mathrm{O}(5) \# 4-\mathrm{K}(1)-\mathrm{O}(1) \# 4$ & $58.91(9)$ & $\mathrm{O}(4)-\mathrm{P}(1)-\mathrm{O}(1)$ & $110.08(17)$ \\
\hline $\mathrm{O}(5)-\mathrm{K}(1)-\mathrm{O}(1) \# 4$ & $121.09(9)$ & $\mathrm{O}(3)-\mathrm{P}(1)-\mathrm{O}(2)$ & $108.60(16)$ \\
\hline $\mathrm{O}(3) \# 4-\mathrm{K}(1)-\mathrm{O}(1) \# 4$ & $50.46(8)$ & $\mathrm{O}(4)-\mathrm{P}(1)-\mathrm{O}(2)$ & $110.61(16)$ \\
\hline $\mathrm{O}(3)-\mathrm{K}(1)-\mathrm{O}(1) \# 4$ & $129.54(8)$ & $\mathrm{O}(1)-\mathrm{P}(1)-\mathrm{O}(2)$ & $106.51(18)$ \\
\hline $\mathrm{O}(4) \# 8-\mathrm{K}(1)-\mathrm{O}(1) \# 4$ & $102.93(8)$ & & \\
\hline \multicolumn{4}{|l|}{$\mathrm{Sr}\left(\mathrm{MoPO}_{6}\right)_{2}$} \\
\hline \multicolumn{4}{|l|}{ Distances $(\AA)$} \\
\hline $\operatorname{Sr}(1)-O(5) \# 1$ & $2.602(2)$ & $\mathrm{Mo}(2)-\mathrm{O}(4) \# 6$ & $1.935(2)$ \\
\hline $\operatorname{Sr}(1)-\mathrm{O}(11) \# 2$ & $2.607(2)$ & $\mathrm{Mo}(2)-\mathrm{O}(1) \# 3$ & $2.046(2)$ \\
\hline $\operatorname{Sr}(1)-\mathrm{O}(3) \# 3$ & $2.664(2)$ & $\mathrm{Mo}(2)-\mathrm{O}(8)$ & $2.108(2)$ \\
\hline $\operatorname{Sr}(1)-O(1) \# 3$ & $2.931(2)$ & $\mathrm{Mo}(2)-\mathrm{O}(7)$ & $2.222(2)$ \\
\hline
\end{tabular}




\begin{tabular}{|c|c|c|c|}
\hline $\operatorname{Sr}(1)-\mathrm{O}(9) \# 2$ & $3.196(3)$ & $\mathrm{P}(1)-\mathrm{O}(10)$ & $1.521(2)$ \\
\hline $\mathrm{Mo}(1)-\mathrm{O}(5)$ & $1.706(2)$ & $\mathrm{P}(1)-\mathrm{O}(7)$ & $1.531(2)$ \\
\hline $\mathrm{Mo}(1)-\mathrm{O}(12)$ & $1.710(2)$ & $\mathrm{P}(1)-\mathrm{O}(4)$ & $1.550(2)$ \\
\hline $\mathrm{Mo}(1)-\mathrm{O}(2) \# 4$ & $1.978(2)$ & $\mathrm{P}(1)-\mathrm{O}(1)$ & $1.551(2)$ \\
\hline $\mathrm{Mo}(1)-\mathrm{O}(9) \# 2$ & $2.055(2)$ & $\mathrm{P}(2)-\mathrm{O}(6)$ & $1.531(2)$ \\
\hline Mo(1)-O(10) & $2.123(2)$ & $\mathrm{P}(2)-\mathrm{O}(8)$ & $1.532(2)$ \\
\hline $\mathrm{Mo}(1)-\mathrm{O}(6) \# 5$ & $2.128(2)$ & $\mathrm{P}(2)-\mathrm{O}(9)$ & $1.540(3)$ \\
\hline $\mathrm{Mo}(2)-\mathrm{O}(3)$ & $1.704(2)$ & $\mathrm{P}(2)-\mathrm{O}(2)$ & $1.541(2)$ \\
\hline $\mathrm{Mo}(2)-\mathrm{O}(11)$ & $1.707(3)$ & & \\
\hline \multicolumn{4}{|l|}{ Angles $\left(^{\circ}\right)$} \\
\hline $\mathrm{O}(5) \# 1-\mathrm{Sr}(1)-\mathrm{O}(11) \# 2$ & $76.57(8)$ & $\mathrm{O}(3)-\mathrm{Mo}(2)-\mathrm{O}(4) \# 6$ & $99.01(11)$ \\
\hline $\mathrm{O}(5) \# 1-\mathrm{Sr}(1)-\mathrm{O}(3) \# 3$ & $92.69(7)$ & $\mathrm{O}(11)-\mathrm{Mo}(2)-\mathrm{O}(4) \# 6$ & $102.09(11)$ \\
\hline $\mathrm{O}(11) \# 2-\mathrm{Sr}(1)-\mathrm{O}(3) \# 3$ & $94.28(7)$ & $\mathrm{O}(3)-\mathrm{Mo}(2)-\mathrm{O}(1) \# 3$ & $93.79(10)$ \\
\hline $\mathrm{O}(5) \# 1-\mathrm{Sr}(1)-\mathrm{O}(1) \# 3$ & $63.80(7)$ & $\mathrm{O}(11)-\mathrm{Mo}(2)-\mathrm{O}(1) \# 3$ & $92.56(10)$ \\
\hline $\mathrm{O}(11) \# 2-\mathrm{Sr}(1)-\mathrm{O}(1) \# 3$ & $132.36(7)$ & $\mathrm{O}(4) \# 6-\mathrm{Mo}(2)-\mathrm{O}(1) \# 3$ & $157.70(11)$ \\
\hline $\mathrm{O}(3) \# 3-\mathrm{Sr}(1)-\mathrm{O}(1) \# 3$ & $63.90(7)$ & $\mathrm{O}(3)-\mathrm{Mo}(2)-\mathrm{O}(8)$ & $162.61(10)$ \\
\hline $\mathrm{O}(5) \# 1-\mathrm{Sr}(1)-\mathrm{O}(9) \# 2$ & $132.50(7)$ & $\mathrm{O}(11)-\mathrm{Mo}(2)-\mathrm{O}(8)$ & $92.24(11)$ \\
\hline $\mathrm{O}(11) \# 2-\mathrm{Sr}(1)-\mathrm{O}(9) \# 2$ & $70.89(7)$ & $\mathrm{O}(4) \# 6-\mathrm{Mo}(2)-\mathrm{O}(8)$ & $85.14(9)$ \\
\hline $\mathrm{O}(3) \# 3-\mathrm{Sr}(1)-\mathrm{O}(9) \# 2$ & $57.25(7)$ & $\mathrm{O}(1) \# 3-\mathrm{Mo}(2)-\mathrm{O}(8)$ & $77.49(9)$ \\
\hline $\mathrm{O}(1) \# 3-\mathrm{Sr}(1)-\mathrm{O}(9) \# 2$ & $118.23(7)$ & $\mathrm{O}(3)-\mathrm{Mo}(2)-\mathrm{O}(7)$ & $89.66(10)$ \\
\hline $\mathrm{O}(5)-\mathrm{Mo}(1)-\mathrm{O}(12)$ & $103.32(12)$ & $\mathrm{O}(11)-\mathrm{Mo}(2)-\mathrm{O}(7)$ & $164.37(10)$ \\
\hline $\mathrm{O}(5)-\mathrm{Mo}(1)-\mathrm{O}(2) \# 4$ & $97.47(10)$ & $\mathrm{O}(4) \# 6-\mathrm{Mo}(2)-\mathrm{O}(7)$ & $84.21(10)$ \\
\hline $\mathrm{O}(12)-\mathrm{Mo}(1)-\mathrm{O}(2) \# 4$ & $97.96(11)$ & $\mathrm{O}(1) \# 3-\mathrm{Mo}(2)-\mathrm{O}(7)$ & $77.65(9)$ \\
\hline $\mathrm{O}(5)-\mathrm{Mo}(1)-\mathrm{O}(9) \# 2$ & $92.69(11)$ & $\mathrm{O}(8)-\mathrm{Mo}(2)-\mathrm{O}(7)$ & $73.89(9)$ \\
\hline $\mathrm{O}(12)-\mathrm{Mo}(1)-\mathrm{O}(9) \# 2$ & $88.89(11)$ & $\mathrm{O}(10)-\mathrm{P}(1)-\mathrm{O}(7)$ & $111.82(13)$ \\
\hline $\mathrm{O}(2) \# 4-\mathrm{Mo}(1)-\mathrm{O}(9) \# 2$ & $166.07(11)$ & $\mathrm{O}(10)-\mathrm{P}(1)-\mathrm{O}(4)$ & $110.53(14)$ \\
\hline $\mathrm{O}(5)-\mathrm{Mo}(1)-\mathrm{O}(10)$ & $164.54(10)$ & $\mathrm{O}(7)-\mathrm{P}(1)-\mathrm{O}(4)$ & $110.02(13)$ \\
\hline $\mathrm{O}(12)-\mathrm{Mo}(1)-\mathrm{O}(10)$ & $90.93(11)$ & $\mathrm{O}(10)-\mathrm{P}(1)-\mathrm{O}(1)$ & $109.54(13)$ \\
\hline $\mathrm{O}(2) \# 4-\mathrm{Mo}(1)-\mathrm{O}(10)$ & $86.25(9)$ & $\mathrm{O}(7)-\mathrm{P}(1)-\mathrm{O}(1)$ & $109.98(13)$ \\
\hline $\mathrm{O}(9) \# 2-\mathrm{Mo}(1)-\mathrm{O}(10)$ & $81.50(9)$ & $\mathrm{O}(4)-\mathrm{P}(1)-\mathrm{O}(1)$ & $104.73(13)$ \\
\hline $\mathrm{O}(5)-\mathrm{Mo}(1)-\mathrm{O}(6) \# 5$ & $90.37(11)$ & $\mathrm{O}(6)-\mathrm{P}(2)-\mathrm{O}(8)$ & $103.46(14)$ \\
\hline $\mathrm{O}(12)-\mathrm{Mo}(1)-\mathrm{O}(6) \# 5$ & $164.91(11)$ & $\mathrm{O}(6)-\mathrm{P}(2)-\mathrm{O}(9)$ & $111.17(13$ \\
\hline $\mathrm{O}(2) \# 4-\mathrm{Mo}(1)-\mathrm{O}(6) \# 5$ & $86.33(9)$ & $\mathrm{O}(8)-\mathrm{P}(2)-\mathrm{O}(9)$ & $110.90(14)$ \\
\hline $\mathrm{O}(9) \# 2-\mathrm{Mo}(1)-\mathrm{O}(6) \# 5$ & $84.09(9)$ & $\mathrm{O}(6)-\mathrm{P}(2)-\mathrm{O}(2)$ & $112.71(13)$ \\
\hline $\mathrm{O}(10)-\mathrm{Mo}(1)-\mathrm{O}(6) \# 5$ & $74.85(9)$ & $\mathrm{O}(8)-\mathrm{P}(2)-\mathrm{O}(2)$ & $111.67(14)$ \\
\hline $\mathrm{O}(3)-\mathrm{Mo}(2)-\mathrm{O}(11)$ & $103.26(12)$ & $\mathrm{O}(9)-\mathrm{P}(2)-\mathrm{O}(2)$ & $107.00(14)$ \\
\hline
\end{tabular}


Table S3. Bond valence sums contributions for $\left[\mathrm{MoO}_{6}\right]^{6-}$ and $\left[\mathrm{PO}_{4}\right]^{3-}$ in $\mathrm{Cs}_{3} \mathrm{Mo}_{12} \mathrm{PO}_{40}$, $\mathrm{LiK}\left(\mathrm{MoPO}_{6}\right)_{2}$ and $\mathrm{Sr}\left(\mathrm{MoPO}_{6}\right)_{2}$.

$\mathrm{Cs}_{3} \mathrm{Mo}_{12} \mathrm{PO}_{40}$

\begin{tabular}{llll}
\hline & $\mathrm{S}_{\mathrm{i}}$ & $\Sigma \mathrm{S}_{\mathrm{i}}{ }^{a}$ & $V-\Sigma \mathrm{S}_{\mathrm{i}}$ \\
\hline$\left[\mathrm{MoO}_{6}\right]^{6-}$ & $\mathrm{Mo-O}(1)$ & 0.95 & 1.05 \\
& $\mathrm{Mo-O}(1)$ & 0.95 & 1.05 \\
& $\mathrm{Mo}-\mathrm{O}(2)$ & 0.24 & 1.76 \\
& $\mathrm{Mo}-\mathrm{O}(3)$ & 0.98 & 1.02 \\
& $\mathrm{Mo}-\mathrm{O}(3)$ & 0.98 & 1.02 \\
$\Sigma \mathrm{S}_{\mathrm{v}}$ & $\mathrm{Mo-O}(4)$ & 1.84 & 0.16 \\
\hline
\end{tabular}

\begin{tabular}{llll}
\hline & $\mathrm{S}_{\mathrm{i}}$ & $\Sigma \mathrm{S}_{\mathrm{i}}^{b}$ & $V-\Sigma \mathrm{S}_{\mathrm{i}}$ \\
\hline$\left[\mathrm{PO}_{4}\right]^{3-}$ & $\mathrm{P}-\mathrm{O}(2)$ & 1.23 & 0.77 \\
& $\mathrm{P}-\mathrm{O}(2)$ & 1.23 & 0.77 \\
& $\mathrm{P}-\mathrm{O}(2)$ & 1.23 & 0.77 \\
& $\mathrm{P}-\mathrm{O}(2)$ & 1.23 & 0.77 \\
$\Sigma \mathrm{S}_{\mathrm{v}}$ & & 4.92 & \\
\hline
\end{tabular}

$\mathrm{LiK}\left(\mathrm{MoPO}_{6}\right)_{2}$

\begin{tabular}{llll}
\hline & $\mathrm{S}_{\mathrm{i}}$ & $\Sigma \mathrm{S}_{\mathrm{i}}^{a}$ & $V-\Sigma \mathrm{S}_{\mathrm{i}}$ \\
\hline$\left[\mathrm{MoO}_{6}\right]^{6-}$ & $\mathrm{Mo-O}(1)$ & 0.79 & 1.21 \\
& $\mathrm{Mo-O}(2)$ & 0.74 & 1.26 \\
& $\mathrm{Mo-O}(3)$ & 0.52 & 1.48 \\
& $\mathrm{Mo-O}(4)$ & 0.47 & 1.52 \\
& $\mathrm{Mo-O}(5)$ & 1.67 & 0.33 \\
$\Sigma \mathrm{S}_{\mathrm{v}}$ & $\mathrm{Mo-O}(6)$ & 1.80 & 0.2 \\
\hline
\end{tabular}

\begin{tabular}{llll}
\hline & $\mathrm{S}_{\mathrm{i}}$ & $\Sigma \mathrm{S}_{\mathrm{i}}^{b}$ & $V-\Sigma \mathrm{S}_{\mathrm{i}}$ \\
\hline$\left[\mathrm{PO}_{4}\right]^{3-}$ & $\mathrm{P}-\mathrm{O}(1)$ & 1.21 & 0.79 \\
& $\mathrm{P}-\mathrm{O}(2)$ & 1.20 & 0.80 \\
& $\mathrm{P}-\mathrm{O}(3)$ & 1.27 & 0.73 \\
& $\mathrm{P}-\mathrm{O}(4)$ & 1.27 & 0.73 \\
$\Sigma \mathrm{S}_{\mathrm{v}}$ & & 4.95 & \\
\hline
\end{tabular}

$\mathrm{Sr}\left(\mathrm{MoPO}_{6}\right)_{2}$

\begin{tabular}{llll}
\hline & $\mathrm{S}_{\mathrm{i}}$ & $\Sigma \mathrm{S}_{\mathrm{i}}{ }^{a}$ & $V-\Sigma \mathrm{S}_{\mathrm{i}}$ \\
\hline$\left[\mathrm{Mo}(1) \mathrm{O}_{6}\right]^{6-}$ & $\mathrm{Mo}(1)-\mathrm{O}(2)$ & 0.82 & 1.18
\end{tabular}




\begin{tabular}{llll}
\hline & $\mathrm{Mo}(1)-\mathrm{O}(5)$ & 1.72 & 0.28 \\
$\mathrm{Mo}(1)-\mathrm{O}(6)$ & 0.55 & 1.45 \\
$\mathrm{Mo}(1)-\mathrm{O}(9)$ & 0.67 & 1.33 \\
& $\mathrm{Mo}(1)-\mathrm{O}(10)$ & 0.56 & 1.44 \\
& $\mathrm{Mo}(1)-\mathrm{O}(12)$ & 1.70 & 0.3 \\
$\Sigma \mathrm{S}_{\mathrm{V}}$ & & 6.02 & \\
\hline
\end{tabular}

\begin{tabular}{llll}
\hline & $\mathrm{S}_{\mathrm{i}}$ & $\Sigma \mathrm{S}_{\mathrm{i}}{ }^{a}$ & $V-\Sigma \mathrm{S}_{\mathrm{i}}$ \\
\hline$\left[\mathrm{Mo}(2) \mathrm{O}_{6}\right]^{6-}$ & $\mathrm{Mo}(2)-\mathrm{O}(7)$ & 0.68 & 1.32 \\
& $\mathrm{Mo}(2)-\mathrm{O}(8)$ & 1.72 & 0.28 \\
& $\mathrm{Mo}(2)-\mathrm{O}(9)$ & 0.93 & 1.07 \\
& $\mathrm{Mo}(2)-\mathrm{O}(10)$ & 0.43 & 1.57 \\
& $\mathrm{Mo}(2)-\mathrm{O}(11)$ & 0.58 & 1.42 \\
$\Sigma \mathrm{S}_{\mathrm{V}}$ & $\mathrm{Mo}(2)-\mathrm{O}(12)$ & 1.72 & 0.28 \\
\hline
\end{tabular}

\begin{tabular}{llll}
\hline & $\mathrm{S}_{\mathrm{i}}$ & $\Sigma \mathrm{S}_{\mathrm{i}}^{b}$ & $V-\Sigma \mathrm{S}_{\mathrm{i}}$ \\
\hline$\left[\mathrm{P}(1) \mathrm{O}_{4}\right]^{3-}$ & $\mathrm{P}(1)-\mathrm{O}(1)$ & 1.20 & 0.80 \\
& $\mathrm{P}(1)-\mathrm{O}(4)$ & 1.20 & 0.80 \\
& $\mathrm{P}(1)-\mathrm{O}(7)$ & 1.26 & 0.74 \\
& $\mathrm{P}(1)-\mathrm{O}(10)$ & 1.30 & 0.70 \\
$\Sigma \mathrm{S}_{\mathrm{v}}$ & & 4.96 & \\
\hline
\end{tabular}

\begin{tabular}{clll}
\hline & $\mathrm{S}_{\mathrm{i}}$ & $\Sigma \mathrm{S}_{\mathrm{i}}^{b}$ & $V-\Sigma \mathrm{S}_{\mathrm{i}}$ \\
\hline$\left[\mathrm{P}(2) \mathrm{O}_{4}\right]^{3-}$ & $\mathrm{P}(2)-\mathrm{O}(2)$ & 1.23 & 0.77 \\
& $\mathrm{P}(2)-\mathrm{O}(6)$ & 1.26 & 0.74 \\
& $\mathrm{P}(2)-\mathrm{O}(8)$ & 1.26 & 0.74 \\
& $\mathrm{P}(2)-\mathrm{O}(9)$ & 1.23 & 0.77 \\
& & 4.98 & \\
${ }^{a} \mathrm{R}_{0} \frac{\mathrm{S}_{\mathrm{v}}}{\left(\mathrm{Mo}^{\mathrm{VI}}-\mathrm{O}\right)=1.907 ;{ }^{b} \mathrm{R}_{0}\left(\mathrm{P}^{\mathrm{V}}-\mathrm{O}\right)=1.617 ;{ }^{a, b} \mathrm{~S}_{\mathrm{i}}=\exp \left[\left(\mathrm{R}_{\mathrm{i}}-\mathrm{R}_{0}\right) / \mathrm{B}\right] .{ }^{1}}$
\end{tabular}




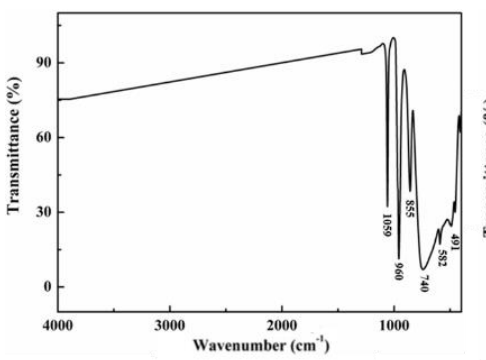

(a)

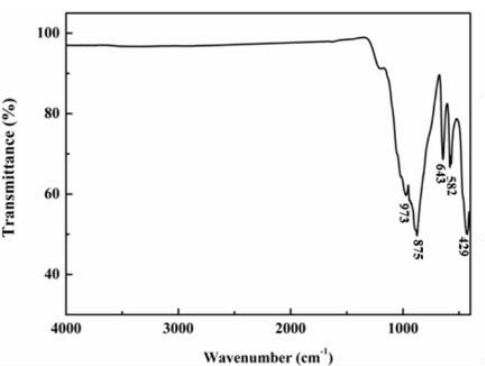

(b)

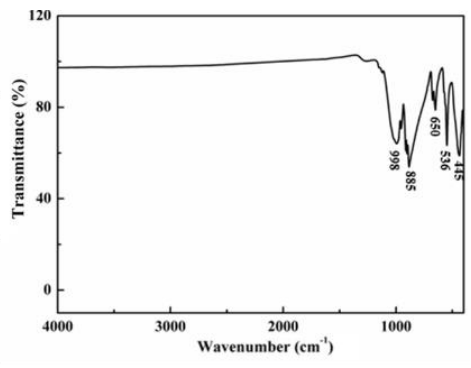

(c)

Figure S1. IR diffuse reflectance spectra of $\mathrm{Cs}_{3} \mathrm{Mo}_{12} \mathrm{PO}_{40}(\mathrm{a}), \mathrm{LiK}\left(\mathrm{MoPO}_{6}\right)_{2}$ (b) and $\mathrm{Sr}\left(\mathrm{MoPO}_{6}\right)_{2}$ (c).

Table S4. The assignments of the infrared absorption peaks for $\mathrm{Cs}_{3} \mathrm{Mo}_{12} \mathrm{PO}_{40}, \mathrm{LiK}\left(\mathrm{MoPO}_{6}\right)_{2}$ and $\mathrm{Sr}\left(\mathrm{MoPO}_{6}\right)_{2}$.

\begin{tabular}{cccc}
\hline Mode description $\left(\mathrm{cm}^{-1}\right)$ & $\mathrm{Cs}_{3} \mathrm{Mo}_{12} \mathrm{PO}_{40}$ & $\mathrm{LiK}\left(\mathrm{MoPO}_{6}\right)_{2}$ & $\mathrm{Sr}\left(\mathrm{MoPO}_{6}\right)_{2}$ \\
\hline$v_{\text {as }}(\mathrm{P}-\mathrm{O})$ & 1059 & $/$ & 998 \\
$v_{\mathrm{s}}(\mathrm{P}-\mathrm{O})$ & 960 & 973 & $/$ \\
$\delta_{\mathrm{as}}(\mathrm{O}-\mathrm{P}-\mathrm{O})$ & 740,582 & 643,582 & 650,536 \\
$\delta_{\mathrm{s}}(\mathrm{O}-\mathrm{P}-\mathrm{O})$ & 491 & 429 & 445 \\
$\mathrm{Mo}-\mathrm{O}$ vibration & 855 & 875 & 885 \\
\hline
\end{tabular}

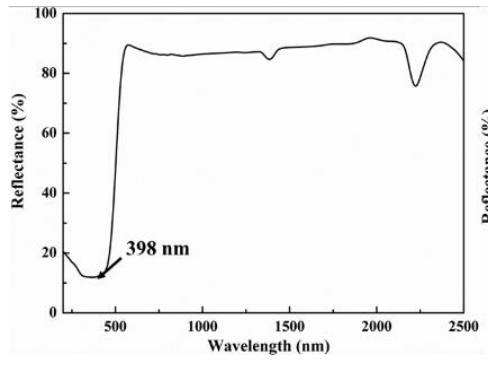

(a)

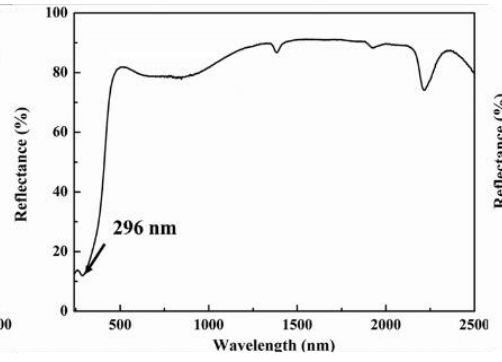

(b)

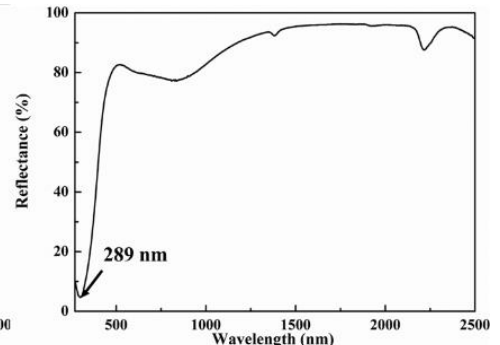

(c)

Figure S2. UV-VIS-NIR diffuse reflectance spectra of $\mathrm{Cs}_{3} \mathrm{Mo}_{12} \mathrm{PO}_{40}(\mathrm{a}), \mathrm{LiK}\left(\mathrm{MoPO}_{6}\right)_{2}$ (b) and $\mathrm{Sr}\left(\mathrm{MoPO}_{6}\right)_{2}(\mathrm{c})$. 


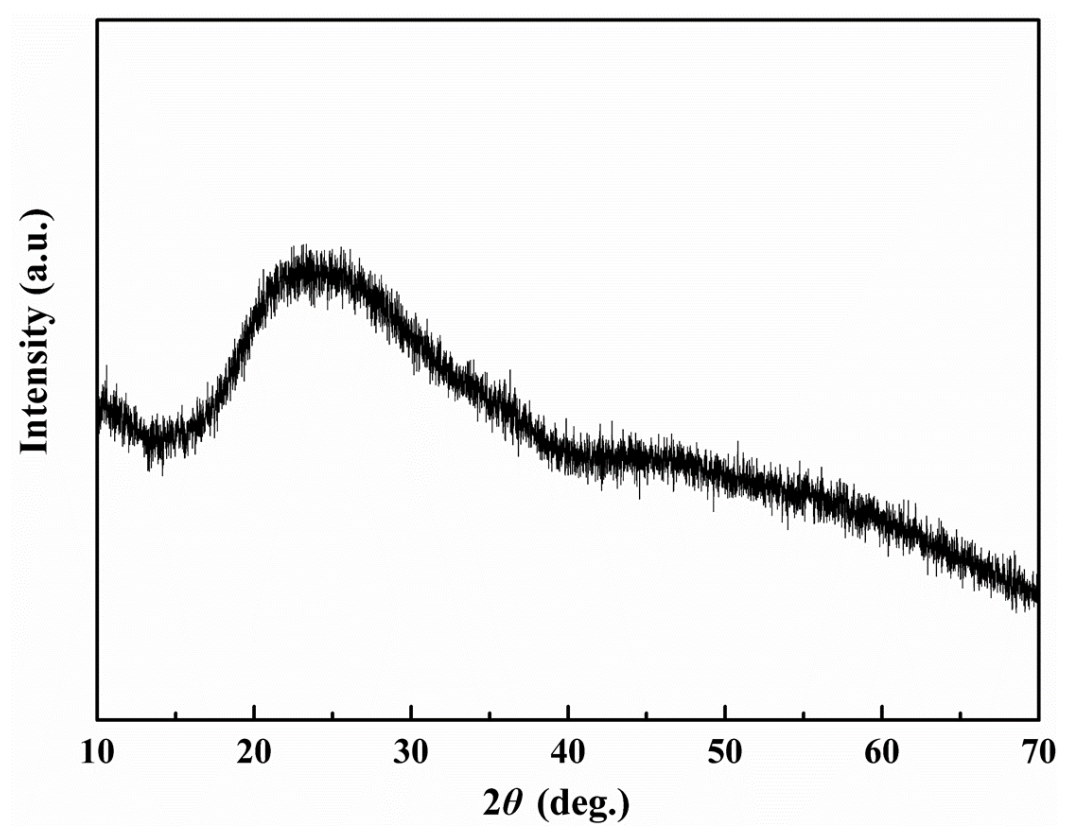

Figure S3. The PXRD patterns of residues after TG/DSC of LiK(MoPO $)_{2}$.

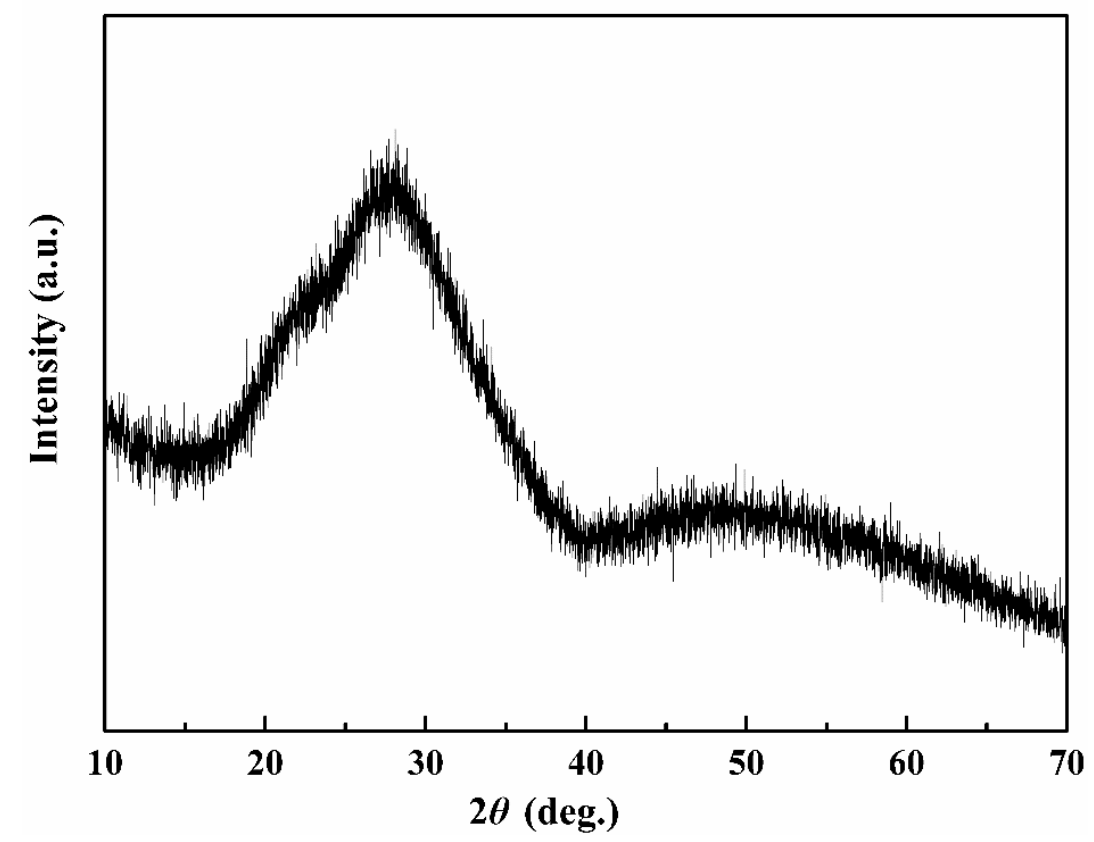

Figure S4. The PXRD patterns of residues after TG/DSC of $\mathrm{Sr}\left(\mathrm{MoPO}_{6}\right)_{2}$.

\section{References:}

1. Brown, I. D.; Altermatt, D. Bond-Valence Parameters Obtained from a Systematic Analysis of the Inorganic Crystal Structure Database. Acta Cryst. 1985, B41, 244247. 